\title{
Entrelacs
}

Cinéma et audiovisuel

Jodorowsky, d'un art à l'autre

\section{Alejandro Jodorowsky et le jeu vidéo : l'occasion manquée}

\section{Thierry Serdane}

\section{(2) OpenEdition}

1 Journals

Édition électronique

URL : http://journals.openedition.org/entrelacs/5665

DOI : 10.4000/entrelacs.5665

ISSN : 2261-5482

Éditeur

Éditions Téraèdre

Référence électronique

Thierry Serdane, «Alejandro Jodorowsky et le jeu vidéo : l'occasion manquée », Entrelacs [En ligne], 16। 2019, mis en ligne le 06 novembre 2019, consulté le 05 décembre 2019. URL : http://

journals.openedition.org/entrelacs/5665; DOI : 10.4000/entrelacs.5665

Ce document a été généré automatiquement le 5 décembre 2019

Tous droits réservés 


\title{
Alejandro Jodorowsky et le jeu vidéo : l'occasion manquée
}

\author{
Thierry Serdane
}

\section{Introduction}

1 Quand, voici quelque vingt ans, je découvrais l'œuvre de bandes dessinées d'Alejandro Jodorowsky, je n'étais pas sensibilisé à son univers, pas plus qu'à un regard sur le jeu vidéo qui puisse valoir de s'y intéresser pour autre chose qu'un passe-temps, certes addictif, mais un passe-temps quand même. De la lecture du premier tome des Technopères ${ }^{1}$ à sa sortie, il ne me reste aucun souvenir qu'elle ait suscité une quelconque réflexion à propos du jeu vidéo. Les états d'âme d'Albino², le protagoniste de la série, n'étaient qu'éléments de l'arc narratif de la fiction. Vingt ans plus tard, alors que réfléchir sur le jeu vidéo était devenu mon métier, la relecture de l'épisode puis de la série complète, se transforma en véritable mise en abîme. Entre temps, « l'affaire ” $^{3} \mathrm{du}$ Dune de Jodorowsky avait été mise au jour et j'avais pu apprécier la quasi-totalité de son œuvre, ses bandes dessinées, ses romans, ses œuvres cinématographiques. Alors que s'organisait ce numéro de revue sur l'œuvre immense de cet artiste, sur sa capacité à explorer toutes formes d'expression, une absence s'imposait, celle du jeu vidéo. Alejandro Jodorowsky n'a peut-être pas fait de jeu vidéo, mais il l'a saisi par les entrailles pour y lire son avenir. Un soir, alors que j'évoquais la possible visite de l'artiste pour assister à l'évènement qui lui était consacré, une amie me dit qu'elle avait eu l'occasion de le rencontrer lors d'une session de travail sur le tarot, il y a quelques années, et me confiait qu'en dédicace de son livre, il lui avait simplement écrit «de mon âme à ton âme ». 


\section{Les Technopères: une critique lucide de l'industrie vidéoludique}

\section{Une lecture perspicace de l'avenir du jeu vidéo}

2 Les Technopères voit son premier tome édité en 1998 aux Humanoïdes Associés. Il sera suivi de sept autres tomes qui composeront un récit se situant dans un univers initié par L'Incal et poursuivi par La Caste des Méta-barons ${ }^{4}$ qui, au travers de perspectives croisées, va enrichir un univers dystopique complexe.

3 Ce qui va nous intéresser ici, c'est le regard particulier que Jodorowsky porte sur les jeux vidéo à travers son introduction comme élément contextuel et diégétique majeur de son scénario. Il ne s'agit pas de tenter une nouvelle exploration de cet univers ou de ses représentations - on peut trouver une très complète et récente analyse ${ }^{5}$ - qui met en évidence la dimension critique du récit comme l'audace nécessaire aux dessinateurs à figurer une "informatique irreprésentable ». À chaque étape du parcours initiatique d'Albino, les références utilisées, les situations mises en scène font écho aux problématiques déjà posées par le jeu vidéo au moment de l'écriture du scénario, mais révèlent peut-être plus encore celles à venir. Impensées par la majorité des joueurs, elles n'étaient pas plus investies par les critiques spécialisées ou par ceux qui, d'un point de vue scientifique, commençaient à percevoir un nouvel objet d'étude ${ }^{6}$. Dans le premier tome de l'intégrale ${ }^{7}$, c'est la question de la déréliction qui est abordée. Albino trouve refuge dans les mondes virtuels, et le rappel de sa mère pour retourner à la réalité de son activité professionnelle dans la fromagerie familiale n'est pas sans rappeler la supplique des mères au moment du repas du soir, quand il s'agit d'extraire leurs adolescents de ces "perversions électroniques ». Mais Albino ne se divertit pas, Albino vit des expériences qui produisent une irrépressible envie d'en créer à son tour. Ce «je veux être créateur de jeux, dans une fabrique pan-techno ${ }^{8}$ » est semblable aux vœux des innombrables étudiants qui investissent les écoles de jeux vidéo aujourd'hui, animés de la volonté créatrice des pionniers du médium. On retrouve la même motivation chez Albino que chez Roberta, Eric, ou les deux John ${ }^{9}$ - celle de l'enfant qui, devant l'illusionniste, les yeux écarquillés, veut lui aussi devenir magicien. Dès l'école, Albino est confronté au crédo imposé aux créateurs de jeux et connaît ses premières désillusions, avec la terrible prise de conscience qu'une autre intention anime l'atelier : « ici, on ne fabrique pas les rêves, on les tue ${ }^{10}$. » Albino, se fait hacker, pourfendeur des «boîtes noires ${ }^{11}$, lieux de secrets et de pouvoir; le jeu c'est de comprendre, de connaître pour espérer exister. Au fil des tomes et de la formation d'Albino, les masques tombent, le parcours initiatique s'assombrit et révèle une vérité cynique : «[...] L'école nous attendait pour nous apprendre à mentir, trahir, tricher, escroquer, voler, exploiter et ruiner les autres...12 ${ }^{12}$ Celui qui résistera, comme un de ses compagnons rebelles qui lancera aux maîtres "[...] je conchie la technique ${ }^{13}$ ", sera châtié tel « un infect rétrotroglosocialik $!^{14}$ » et verra ses pieds sectionnés, inutiles à sa nouvelle fonction. Plus tard, alors qu'il est présenté par son double négatif ${ }^{15}$ à un nouveau maître virtuel, un échange nourri ${ }^{16}$ à propos de sa condition va s'engager :

- [Le maître virtuel] Dites-moi vermisseaux, pourquoi êtes-vous venus jusqu'ici ?

- [Albino] Pour apprendre à créer de beaux jeux, maître.

- [Le double négatif] Mais non, crétin, nous sommes ici pour apprendre à les vendre!

- [Le maître virtuel] Exactement gamins, avant de créer des jeux, il faut savoir qui 
va les acheter et comment les embobiner pour qu'ils viennent se prendre dans nos filets de vente [...]. Nous devons profiter de leurs défauts et de manière détournée, subliminale, les encourager... [...] Plus il sera un raté, plus le citoyen achètera nos jeux pour se fabriquer artificiellement une vie de jouissance et de triomphes...

- [Albino] Je ne veux pas! Je ne peux pas le croire, la création de jeu ne peut être aussi abjecte!

Pour autant Albino devra s'y résoudre.

5 Si le trait peut paraître forcé, les approches prônées dans la réalité par les gourous du neuromarketing comme celles enseignées dans les formations au game-design de la monétisation dans les jeux freemium ${ }^{17}$, montrent qu'il l'est à peine. Nous y reviendrons. Ce qui est plus étonnant, c'est la perspicacité de Jodorowsky sur le devenir d'une industrie qui, si elle avait déjà traversé de nombreuses crises, n'était pas encore perçue comme aussi perverse.

\section{Une dimension politique identifiée}

Dans une sorte de prédiction auto-réalisatrice, Jodorowsky rappelle à chaque début de chapitre, la tension téléologique qui anime Albino, une fois devenu technopère : «Le projet d'une nouvelle société où les relations humaines sont plus importantes que les développements technologiques». Cette opposition entre sociétés humaine et technologique, l'auteur des Technopères l'expose depuis L'Incal - pourquoi passer par cet objet trivial, les jeux vidéo, pour illustrer une nouvelle fois cette thèse? Peut-être, parce que Jodorowsky a compris bien plus tôt que d'autres la puissance de ce médium, tant pour agir sur les peuples tel un nouvel opium, qu'en tant que révélateur de la nouvelle matrice de pouvoir qui se développe avec la cybernétique et la postmodernité, une société de contrôle appuyée sur sa propre déconstruction. Comment sinon, expliquer cette violence des propos: «leurrer, disséquer les faiblesses du genre humain, profiter des maux de l'âme pour vendre des jeux qui avaient toutes les propriétés d'une drogue [...]. L'art est un commerce de plus, rien d'autre. Nous devons apprendre à exploiter la névrose de nos clients. » Jodorowsky voit dans le jeu vidéo qu'il met en scène, l'arme absolue de la société dystopique qu'il décrit. Les armes létales qui rivalisent de puissance de scène en scène, ne sont pas suffisantes à l'expression du pouvoir, et le recours à ce soft power, à la force de l'illusion pour dominer les masses est proprement significatif d'un changement de paradigme.

\section{Une écriture documentée}

7 Jodorowsky n'écrit pas n'importe quoi pour alimenter son univers. Si nombre d'arcs scénaristiques sont fantasmés, nourris par toute la culture de science-fiction qu'il a également participée à créer, son propos sur les technologies mises en œuvre, sur les objets, leurs formes, leurs fonctions, sur les mots utilisés, montrent sinon une connaissance de l'informatique, a minima une proximité avec ce phénomène.

Il fait par exemple référence à un processeur « 5040 » que seuls des contemporains investis de la lutte animant Motorola et IBM/INTEL dans les années 1980/1990 peuvent identifier ${ }^{18}$. Les pratiques de l'industrie concernant l'organisation de play tests, c'est-àdire de tests effectués par des groupes de joueurs qui servent de base à l'évaluation de la réception d'un jeu, sont inconnues du grand public et pas même évoquées par les médias spécialisés à l'époque où le scénario est écrit. La liste est longue des références 
qui attestent soit d'une pratique éclairée du milieu de la création de jeux vidéo, soit d'un investissement documentaire important. Mais c'est bien évidemment la mise en perspective de l'informatique en général et du jeu vidéo en particulier qui étonne, tant celle-ci est pertinente. Dans cette deuxième moitié des années 1990, la recherche en sciences humaines et sociales à propos du jeu vidéo, comme la réflexion critique, est balbutiante. Peu s'intéressent à ces questions, et si la critique des industries culturelles comme des médias est très présente depuis Théodor W. Adorno, Max Horkheimer, puis

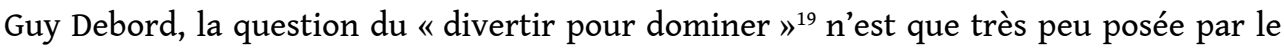
jeu vidéo. Seul, peut-être l'ouvrage de Pierre Bruno paru en $1993^{20}$ aborde le sujet pour conclure finalement qu'il n'y a pas vraiment de différence avec la question bien plus ancienne de l'influence du conte. Il faudra attendre le début des années 2000 pour que la dimension psycho-socio-politique du jeu vidéo soit questionnée de façon plus significative.

\section{Albino le jeune ou la maturité du jeu vidéo}

\section{Le jeu vidéo entre production industrielle et création indépendante}

9 Comme toute production de ce que l'on doit désormais nommer industries culturelles et créatives, le jeu vidéo est l'objet de tensions. La tension entre créateurs et vendeurs, entre artistes et industrie, entre rêveurs et gestionnaires, est personnifiée par Albino à chaque étape de son parcours, à chaque désillusion, à chaque rébellion: de son affirmation « je ne veux pas être un marchand d'immondices, je veux être un créateur de mondes merveilleux ", jusqu'à la mention du projet mainstream, encore innommé qu'il subira indirectement, " ...pendant que ton Toulkou [son double asservi] apprendra à mondialiser les goûts, uniformisant les mentalités et les comportements... »

10 C'est toute l'histoire du jeu vidéo, comme industrie, mais aussi comme potentialité contre-culturelle qui est évoquée. Une industrie contemporaine de celle de l'informatique, qui souvent la suit, mais parfois la précède, l'initie. Au début des années 1960 , les premiers rêveurs, vont "hacker» une informatique industrielle issue du projet cybernétique. Ce projet d'un nouvel humanisme initié par Norbert Wiener en $1948^{21}$ est une réaction à la barbarie résultant de l'incapacité de l'homme à lutter contre sa perte, à réguler son cycle et son entropie. C'est le manque de maitrise de l'information que le projet cybernétique pointe du doigt, et la machine à information en devient un des moyens. Mais le nouveau paradigme prend assez vite la forme d'une religion du contrôle. Les premiers hackers, ceux du Massachussetts Institut of Technologie (MIT), les hackers de code, comme la génération suivante, celle du hack matériel dans les années 1970, vont initier un contre-projet: celui de l'informatique personnelle ${ }^{22}$. Un projet porteur de valeurs, de partage, d'émancipation, qui sera autant animé, qu'illustré par deux motivations, l'art et le jeu. Ces deux modes de hacking porteront un coup dur mais pas fatal à ce que l'on peut nommer «la logique du terminal $»^{23}$, cette logique qui par le contrôle et le seul accès aux terminaux, centralise le pouvoir et les utilisateurs des systèmes d'information. Mais née dans une période qui va promouvoir des valeurs libertariennes où le libéral prendra vite le pas sur le libertaire, l'utopie va se transformer en nouvelle guerre commerciale dans l'immense marché des ordinateurs personnels, du logiciel et des jeux vidéo. Un temps encore, les 
ingénieurs rêveurs, les auteurs visionnaires pourront exister sur ce marché, mais très vite le poids économique, les contraintes du mainstream, rendront compliqué l'accès aux gondoles de supermarchés ou aux chaînes spécialisées. Avec la dématérialisation, le développement des boutiques en ligne et la disponibilité d'outils de création de plus haut niveau ${ }^{24}$, moins exigeants du point de vue de la compétence informatique, favorisèrent l'émergence puis la saturation de l'offre de jeux vidéo. Le jeu vidéo est devenu un médium a priori accessible du point de vue de sa production. L'effort de formation qu'il nécessite pour exister sur le marché est semblable à celui qu'exige d'autres formes de présences médiatiques contemporaines, qu'il s'agisse des musiques actuelles, des Web TV ou de l'édition numérique. Mais là comme ailleurs, la facilité d'accès ne fait pas la qualité, et la visibilité reste plus un problème de marketing que de qualité intrinsèque du jeu. D'autres problématiques se posent, et la première d'entre elles est que la dématérialisation porte en elle le piège originel, puisqu'elle conduit inexorablement à retrouver "la logique du terminal», le retour de la boîte noire, l'abandon du pouvoir aux maîtres de l'accès. Il reste que l'élan donné par cette possibilité d'exister comme indépendant dans cet univers du jeu vidéo - indépendant économiquement, sans contrainte d'un éditeur, indépendant parce que libre de contrainte de support, d'outil de diffusion, indépendant parce que créateur sans objet économique, animé par le seul projet de dire, de montrer, d'émanciper, d'émouvoir est un moteur qui rend possible l'utilisation de ce médium, comme Albino y aspire dans la fiction de Jodorowsky. Cette maturité possible du jeu vidéo s'exprime aujourd'hui dans de nombreuses niches, dans l'art-game, le game-art, le news-game, le political-game ${ }^{25}$. Le jeu vidéo traverse la société et la société traverse le jeu vidéo.

\section{L'immersion au risque de l'addiction}

11 Une autre tension, plus subtile, est également évoquée, tant par le texte que par l'image dans Les Technopères. Une tension qui oppose ou lie immersion et addiction. L'immersion peut prendre des formes multiples et chaque forme d'art - mais c'est aussi le cas d'activités qui ne revendiquent aucune dimension artistique - en fait une description singulière. Dans le jeu vidéo, ce concept a longtemps été le concept majeur, celui qui était cité par la presse spécialisée comme témoin de la qualité d'un jeu, celui qui représentait un «Graal» à atteindre pour les concepteurs, celui qui sans être nommé, définissait l'expérience décrite par les joueurs qui la rencontraient. Aujourd'hui, s'il reste le concept de référence pour de nombreux jeux, la multiplicité des formes, la casualisation ${ }^{26}$ des pratiques, et la prédominance d'autres concepts plus porteurs dans une société " gamifiée ", comme le fun ou le flow, tendent à en atténuer la prédominance. Les jeux qu'Albino veut réaliser sont ceux qui favorisent l'immersion, qui doivent produire des expériences inoubliables pour ceux qui vont les expérimenter. La trace que ces expériences devront laisser doit être positive, émancipatrice. En revanche, les jeux produits par les Technos ont d'autres finalités : divertir, abrutir, pour empêcher toute pensée critique, ou piéger par une immersion dont l'effet psychotrope aboutira à une addiction propice à la soumission. La trace portera alors les marques du conditionnement.

12 Encore une fois la vision est perspicace et anticipatrice et d'une certaine manière la fabrique du jeu vidéo aujourd'hui opère souvent sur les mêmes registres. D'une part des "bâtisseurs de rêves", d'autre part des "dealers". Le terme est fort et bien sûr provocateur, mais comment qualifier certaines pratiques rencontrées dans ce secteur 
d'activité, qui jusque dans les conseils des experts patentés orientent vers ces méthodes. Sur le site de l'Agence Française pour le Jeu Vidéo (AFJV), on trouve notamment les rubriques de Pascal Luban ${ }^{27}$, un consultant réputé, ex-level et game designer dans de très gros studios internationaux, qui s'est aujourd'hui spécialisé entre autres dans l'analyse de la monétisation des jeux freemium ${ }^{28}$. À la lecture de quelquesuns des conseils avisés de l'expert, le constat est significatif. Par exemple, on trouve une entrée dans le sommaire de la formation renvoyé en lien qui s'intitule «Le moteur des jeux freemium : La frustration ». Extrait :

C'est elle qui incite le joueur à dépenser de l'argent dans un jeu «gratuit " (c'est l'auteur qui met entre guillemet). Le mécanisme est simple : 1) Faite en sorte que le joueur apprécie ses premières parties ou premières heures de jeu, 2) donnez-lui l'envie de progresser en lui soumettant des objectifs et en le récompensant pour les avoir atteint, 3) multipliez les objectifs à atteindre ET rendez les progressions pour les atteindre de plus en plus longues. Lorsqu'il ne pourra plus supporter l'attente, le joueur commencera à « acheter $»^{29}$.

13 Le modèle de réussite est construit sur les mêmes ressorts que ceux du marché des jeux d'argent ou de celui des différents trafics de drogue. C'est l'organisation d'une frustration qui ne peut être assouvie que par l'acte d'achat. La méthode assumée comme telle est relativement récente, contemporaine de l'explosion des réseaux sociaux et des smartphones, soit plus d'une dizaine d'années après l'écriture des Technopères. Si le jeu vidéo était déjà une industrie, si l'immersion était recherchée et que bien sûr, les concepteurs comme leurs éditeurs espéraient capter et fasciner leur public jusqu'à leur faire perdre toute notion de temps, la démarche restait identique à celle de la littérature ou du cinéma - laisser la trace durable d'une expérience de qualité dont on se souviendra. L'objectif ici est de toute autre nature - fabriquer une frustration d'ordre addictif avec un cynisme méthodique, et c'est bien ce qu'avait perçu Jodorowsky.

\section{Projection fantasmée plutôt que cible à toucher}

14 La création de jeux vidéo selon Jodorowsky oppose rêveurs et manipulateurs, et le potentiel du médium agirait comme un pharmakon avec une lutte de pouvoir dont l'issue laisse peu de doute. Si ce regard sur le jeu vidéo date de la fin des années 1990, Jodorowsky ne semble pas plus enthousiaste dans les déclarations qu'il fait à ce sujet dans une interview en $2008^{30}$. Pourtant, entre temps, de nombreux créateurs indépendants se sont saisis du jeu vidéo pour donner leur vision du monde, exprimer leurs émotions, dénoncer ce qui leur paraissait abject dans les politiques menées par les puissants ou les modèles économiques imposés par la financiarisation. On pense en particulier aux œuvres de Gonzalo Frasca ou celles du collectif de la Molleindustria. D'autres en ont déconstruit les structures industrielles pour produire en contrepoint des poétiques singulières. Rhétorique procédurale, narration environnementale, distanciation brechtienne, représentent autant d'approches qui s'éloignent des modèles de productions plébiscités et présentés comme incontournables pour satisfaire aux règles d'un marché mainstream, seul garant de réussite dans une industrie globalisée. Ces tentatives sont souvent militantes, ou s'inscrivent dans une démarche proche de mouvements artistiques passés, elles s'expriment au travers de manifestes, se contentent de dénoncer ou tentent même d'émanciper et montrent quoi qu'il en soit qu'elles prennent ce médium au sérieux quant à sa capacité d'expression. Une fois évacuée la notion de «marketing de cible», qui reste d'ailleurs commune aux 
différents marchands culturels - la littérature, la musique et le cinéma ne différent en rien du jeu vidéo sur ce point -, les créateurs de jeux peuvent eux aussi revendiquer une position d'auteurs. Certes, les méthodologies de conception qui ont cours dans le jeu vidéo sont problématiques. Issues du design, elles contraignent le processus de création par des protocoles de construction des idées conditionnés par les possibilités techniques et économiques, mais surtout dans un carcan technologique guidé par des préceptes d'efficience. Pour les concepteurs qui se revendiquent auteurs, qui se ressentent artistes, le chemin est difficile, comme celui d'Albino. Leurs intentions ne visent aucune cible, elles ne sont que fantasmes d'expériences, messages militants, poésie - difficile de convaincre, étude de marché en main, ça n'a pas de sens - la mesure de la réception n'est pas un critère.

\section{Et si... Jodo s'était saisi du médium}

\section{Du roman au film, de la BD au jeu vidéo}

15 En 1973, quand Jodorowsky entreprend non pas d'adapter le Dune de Franck Herbert mais de mettre à l'épreuve sa propre fantasmagorie de l'œuvre, il signe certainement un des actes fondateurs des possibles de la science-fiction au cinéma. Si les quatre années de pré-production et de travaux préparatoires n'aboutiront pas à une sortie en salles, et peuvent résonner alors comme un échec, le talent et l'imagination des artistes associés à l'aventure ne seront pas stériles pour autant et la plupart des œuvres qui suivront en porteront la trace. Comme le dit Jodorowsky, «le projet fut saboté à Hollywood. Il était français et non américain. Le message n'était pas "assez Hollywood" ${ }^{31}$. » C'est le même destin que failli connaître le studio de jeux vidéo $\mathrm{Cryo}^{32}$ à la fin des années 1980. Philippe Ulrich co-fondateur du studio, s'était lui aussi vu confier les droits d'adaptation de l'œuvre d'Herbert par Virgin qui venait de les acquérir pour une adaptation en jeu vidéo. Si l'interprétation de Cryo ne s'accordait pas autant de liberté que la vision qu'en avait eue Jodorowsky, le simple respect de l'atmosphère et des messages du roman, la tentative d'en faire une « œuvre » vidéoludique, et de profiter de la technologie naissante du CD-ROM pour donner de l'espace à la dimension graphique et sonore, en faisaient un objet contre-culturel pour l'industrie du jeu vidéo. La réponse ${ }^{33}$ faite par l'éditeur à l'issue des premières démonstrations fut sans appel : ce n'était pas un jeu vidéo, car il était trop lent, trop verbeux et surtout ça ne répondait pas aux attentes et aux goûts du public américain. Finalement, le studio poursuivra le développement, comme un défi, sans autorisation, pour qu'à l'arrivée la qualité soit malgré tout reconnue du bout des lèvres et le jeu édité. À ce moment-là seulement, Cryo découvrit que Virgin avait confié la réalisation d'un autre Dune à un studio américain, Westwood, qui pour l'occasion inaugura un des tous premiers jeux de stratégie en temps réel, Dune 2, à mille lieues de l'univers d'Herbert mais aussi de Dune. Le jeu de Cryo était subversif sur bien des points au niveau de l'expérience proposée qui s'écartait de la direction prise par l'industrie, mais surtout dans la façon de reconnaître la qualité d'artistes aux membres de l'équipe, tous présentés dans la notice à force de biographies et d'intentions, une sorte de pied de nez d'un droit d'auteur impossible au pays du copyright.

16 La bande dessinée s'impose également dans l'univers du jeu vidéo comme une évidence. Il est plus fréquent de voir des univers de jeux vidéo adaptés à la BD que l'inverse, 
chaque succès qui s'installe dans le temps à l'image de l'univers des Assassin's Creed, ou celui de World of Worcraft suit la tendance, mais dans un cas comme dans l'autre les réussites sont rares. Les mondes du jeu vidéo semblent pauvres une fois mis en cases, et souvent c'est tout simplement qu'ils le sont vraiment. La part du scénario dans ces titres à succès est généralement réduite au strict nécessaire pour justifier l'action. Sans l'interactivité qui offre au joueur d'écrire sa propre histoire, l'aventure tourne court. À l'inverse, ceux de la bande dessinée y apparaissent stéréotypés, articulés autour des seules situations s'accordant avec des "gameplays " éprouvés comme efficaces et en même temps attendus. Assez peu d'inventions finalement. Reste que quand les artistes se saisissent vraiment de ces adaptations, ils arrivent à offrir une véritable et nouvelle expérience esthétique. On peut penser à la prise en charge du médium par Benoit Sokal avec L'Amerzone et la serie des Sibéria ${ }^{34}$, l'influence de la patte graphique de Mœbius sur de nombreux projets, ou encore la très bonne adaptation du comics The Walking Dead par le studio Teltales ${ }^{35}$. Et si le vieux point and click $^{36}$ semble être le pont entre les deux modes d'expressions, il laisse présager du meilleur pour la sortie prochaine de la BD Blacksad par le studio Pendulo.

\section{La question technique}

17 Pour Jodorowsky, le problème avec les jeux vidéo est pour partie lié à ceux qui le font. À la question qui lui était posée lors de cette interview de 2008 , «Le jeu vidéo n'est pas un média que vous souhaiteriez investir? ", il répondait la chose suivante :

18 J'ai tenté d'adapter La Caste des Méta-Barons. Mais le jeu vidéo, comme celui des effets spéciaux numériques au cinéma, est un monde de techniciens. Les techniciens n'ont pas bon goût, ce ne sont pas de grands artistes. Les films d'animation en 3D par exemple sont d'un goût atroce. Tout le monde n'a pas la poésie de Hayao Miyazaki. Bien qu'aujourd'hui ce soit un monde de techniciens, je ne doute pas qu'un jour un artiste parviendra à s'y exprimer ${ }^{37}$.

La réponse est un peu rapide. Difficile de penser que l'acuité de Jodorowsky à analyser le potentiel de ce médium et les tensions qui y opèrent ne l'amène pas à plus de nuances, en séparant, par exemple, les individus de leurs fonctions. Ni la technique, ni l'approche technicienne n'ont de goût, mais l'individu peut traiter artistiquement un médium qui se fera œuvre. Tout matériel ou matériau nécessaire à une expression artistique ou auctorielle est soumis à la maîtrise technique de celui qui les manipule ou en envisage la mise en œuvre. Les matériaux et le matériel qui participent à fabriquer un jeu vidéo, ne sont pas très différents de ceux qu'utilisent le bédéaste, le réalisateur, le peintre, le sculpteur, le musicien ou même l'écrivain. Avec plus ou moins d'incidence, la technique est toujours présente dans le processus de création. Complice ou ennemie, elle reste la compagne de l'artiste et, comme pour Étienne Souriau ${ }^{38}$ qui prêtait différents modes d'existence à l'œuvre, l'individu peut lui aussi passer du technicien à l'artiste. Le philosophe s'intéressait d'un même mouvement à la manière d'exister des matériaux comme des hommes. Gilles Deleuze, dans une célèbre conférence donnée à la FEMIS en 1987, posait sa fameuse question "Qu'est-ce qu'avoir une idée en cinéma? ", à laquelle il répondait que cela consistait à inventer des blocs de mouvements-durée, comme avoir une idée en peinture consisterait à inventer des blocs de lignes-couleurs. Cette proposition s'inscrivait dans une réflexion plus large sur l'acte de création. En ressortait notamment quelques lignes de force, comme celle qu'une idée en général 
n'existait pas, que les idées «sont des potentiels déjà engagés dans tel ou tel mode d'expression et inséparable du mode d'expression ${ }^{39}$ " mais aussi que celui qui a une idée dans un domaine, l'a en fonction des techniques qu'il connait. Ici, Gilles Deleuze fait de l'acte de création une activité partagée par toutes les disciplines mais singulière et spécialisée dans chacune d'entre elles. Dans son interview de 2008, Jodorowsky nous dit qu'il a tenté d'adapter La Caste des Méta-barons en jeu vidéo avant d'abandonner, parce que le jeu vidéo était une affaire de techniciens et que ceux-ci manquaient de sensibilité artistique. Pour Deleuze, l'adaptation n'est pas du fait de celui qui crée l'œuvre originale mais bien de celui qui crée la nouvelle œuvre. Surtout, Deleuze fait la démonstration par l'exemple en convoquant Kurusawa et Dostoïevski pour évoquer la nécessité d'une affinité, d'une familiarité entre l'idée en littérature et l'idée en cinéma pour qu'elles rentrent en résonnance ${ }^{40}$. Alors, Jodorowsky se trompe-t-il? Non, juste que l'adaptation ne se commande pas. Pour adapter une œuvre comme La Caste des Méta-barons en jeu vidéo, il faut avoir une idée en jeu vidéo qui résonne avec l'idée en bande dessinée de leur auteur ou plus largement encore avec les idées qui font que les bandes dessinées de Jodorowsky sont du Jodorowsky. Le processus est pourtant au cœur même de cette expression artistique tant on attend que le dessin et le scénario y soient en familiarité. Pour que Jodorowsky adapte une de ses créations en jeu vidéo, il faut qu'il ait tout simplement les idées de Jodorowsky, mais des idées de Jodorowsky en jeu vidéo.

\section{La VR comme médium d'états de conscience}

Mais peut-être qu'avant d'adapter une des œuvres passées, il s'agit de trouver un champ d'expression digne d'intérêt pour l'artiste. Si les techniciens du jeu vidéo manquent de fibre artistique, certaines technologies qui s'y développent offrent aujourd'hui des perspectives qui ouvrent à de nouvelles expressions fantasmagoriques. Les technologies de réalité augmentée par exemple sont certainement les meilleures alliées des futures expériences ubiquitaires, où la réalité devient l'environnement même d'une fiction élaborée par la palette de traces signifiantes savamment positionnées. Le jeu Pokemon $\mathrm{Go}^{41}(2016)$ a popularisé ces expériences, mais l'ensemble des applications muséales en dévoile d'autres registres. Pour l'auteur du jeu, manipuler ces formes indicielles d'un nouveau type permet tout autant de complexifier que de brouiller les points de vue, les cadres de références, ajouter mais aussi soustraire en contredisant une lecture évidente. Une autre technologie fait son énième come-back, chaque fois trop optimiste, toujours pleine de promesses qui ne rencontrent aucun usage. La réalité virtuelle, celle symboliquement représentée par ces casques protubérants, est à nouveau de retour depuis quelques années. Bien sûr, elle apparaît toujours comme balbutiante, faute de la rencontre d'un public assez large, d'un modèle économique garant d'un marché suffisant pour que les producteurs de contenus s'y engagent pleinement. Ce n'est pas faute de parrains de poids, avec notamment le rachat d'Occulus par Facebook, l'engagement de Sony, Microsoft et du géant des boutiques de jeux en ligne Valve, avec un des casques les plus aboutis en collaboration avec HTC. Mais cette fois-ci, la réalité virtuelle semble avoir vaincu ses démons. Sans être la révolution annoncée, le secteur se développe. L'accès par l'intermédiaire des smartphones et des cardboards ${ }^{42}$ ouvre à l'accès populaire et même si l'expérience n'est pas du même niveau, elle étonne, et suscite la curiosité. À côté de ce premier accès, les modèles hight tech se succèdent avec leurs chapelets d'accessoires qui viennent 
renforcer une expérience de plus en plus riche en sensations. Tapis de marche, gants haptiques, combinaisons sensorielles, dispositifs d'atténuation de la cinétose ${ }^{43}$. Cette recherche d'expérience totale au niveau des sens n'est pas un fantasme contemporain, et sans remonter à l'allégorie de la caverne dans la République de Platon, le projet de Morton Heilig au milieu des années 1960 peut être considéré comme celui qui en fixe l'orientation. Morton Heilig, avec son Sensorama ${ }^{44}$ ne propose rien de moins qu'une machine multisensorielle. Le spectateur est en partie entouré d'un caisson où il peut profiter du film en stéréoscopie et la clôture effectuée d'avec la réalité est renforcée par divers artifices auditifs comme olfactifs.

21 Les nouveaux dispositifs de réalité virtuelle rendent possible de multiples expériences au sein des laboratoires comme au sein des ateliers où des artistes contemporains s'en saisissent. Ils sont propices à fabriquer des «véhicules expérientiels ». Le concept ne qualifie pas l'objet d'usage une fois réalisé mais bien la façon dont il est pensé initialement et, dans ce cas précis, en dehors de tout processus d'idéation propre au design mais bien guidé par le seul fantasme de l'expérience qui sera vécue, comme celle qui préside par exemple à la fabrication d'un ornithoptère ${ }^{45}$, le fantasme du vol animal. Quant à faire l'expérience de la découverte de ses chakras par le jeu vidéo comme Alejandro Jodorowsky le suggère comme un défi ${ }^{46}$... Ces nouveaux dispositifs semblent en tout cas s'imposer pour explorer de nouvelles sensations, de nouvelles perceptions et ouvrir à de nouvelles expressions artistiques.

\section{Conclusion}

Avec Les Technopères, Alejandro Jodorowsky introduit au cœur des années 1990, la question très peu présente encore à propos de ce médium, du divertissement comme instrument de domination. Il ne s'agit plus du " panem et circenses $»^{47}$ de Juvénal, de contrôle social de masse, mais bien de l'utilisation plus subtile des propriétés psychotropes des jeux vidéo au service d'une manipulation des individus. Le concepteur de jeux y apparaît dans toutes ses contradictions, selon qu'il endosse l'habit $\mathrm{du}$ chamane, apte à fabriquer de nouvelles réalités, ou celui du gourou guidé par sa propre folie ou au service d'une société de contrôle. La perception de cette tension, la projection fictionnelle qui en est faite, montrent que Jodorowsky reconnaît la puissance potentielle de ce médium. Si dans son discours, il semble le fustiger, ce n'est peut-être que la déception de ne pas en trouver les clés, de constater que son écosystème n'est pas encore prêt pour qu'il s'en saisisse, que sa grammaire est encore trop pauvre pour qu'elle vaille qu'il la réinvente. Mais le jeu vidéo a évolué : avec l'explosion du jeu vidéo indépendant et même si la reconnaissance institutionnelle d'un jeu d'auteur n'atteint pas encore celle qui permet au cinéma d'exister en dehors des blockbusters hollywoodiens, il est envisageable d'explorer de nouvelles pistes comme ont pu le faire des studios comme Tale of Tales ${ }^{48}$ par exemple. Les technologies de réalités virtuelles, après de multiples tentatives, semblent s'installer dans un régime régulier d'évolution qui promet une dimension immersive de grande qualité et devrait commencer à se démocratiser, mais il leur manque peut-être encore qu'une folie s'en empare. Alors monsieur Jodorowsky, « de votre âme à notre âme », ayez des idées en jeu vidéo. 


\section{NOTES}

1. Alejandro Jodorowsky (scénariste), Zoran Janjetov, Fred Beltran (dessinateurs), Les Technopères, Tome 1: La Pré-école Techno, Les Humanoïdes Associés, Paris 1998, premier des huit tomes de la série.

2. Albino, enfant d'une mère violée et vengeresse se réfugie dans les jeux vidéo et souhaite plus que tout en créer à son tour.

3. Le grand public ne découvre le projet avorté de Jodorowsky qu'à travers le documentaire de Frank Pavich, Jodorowsky's Dune, réalisé en 2013.

4. Alejandro Jodorowsky (scénario), Mœbius (dessin), L'Incal, Début de publication dans le journal Métal Hurlant (1980) puis sous forme d'albums (six tomes), L'Incal - Une aventure de John Difool, Les Humanoïdes Associés, Paris, 1981-1988; Alejandro Jodorowsky, Juan Gimenez, La Caste des MétaBarons (huit tomes), Les Humanoïdes Associés, Paris, 1992-2003.

5. Voir notamment Éric Villagordo, «Les Technopères: la carte informatique, un chemin initiatique », ReS Futurae [en ligne], "Imaginaire informatique et science-fiction », n¹0, 29 novembre 2017, URL : http://journals.openedition.org/resf/1153.

6. En France, la recherche sur l'objet jeu vidéo dans les années 1990 se limite à une petite poignée d'auteurs et porte principalement sur sa description et son analyse.

7. Intégrale en un seul volume, Les Humanoïdes Associés, 2013, p. 11.

8. Ibid., p. 13.

9. Roberta Williams, Eric Chahi, John Romero et John Carmack, grands innovateurs des débuts du jeu vidéo.

10. Ibid., p. 17.

11. Entendre ici, tout dispositif technologique ou structurel opaque, dont les règles qui gèrent ses entrées et ses sorties ne sont connues que de certains, qui gardent ainsi par cette connaissance un pouvoir sur les seuls usagers de ces dispositifs.

12. Ibid., p. 59.

13. Ibid., p. 60.

14. Ibid.

15. Quand Albino, malgré son potentiel, montre quelques signes de fragilité, une sorte de mauvais génie surgira pour l'accompagner, issu de son «techno-esprit " "honteusement refoulée depuis toujours : un égo primitif, cruel et égoïste ».

16. Ibid., p. 80-82.

17. Dans le domaine du jeu vidéo, est identifié comme freemium une production diffusée gratuitement pour ensuite appuyer son modèle économique sur le pari d'une transformation de cet accès gratuit en achats qui peuvent prendre la forme de micro-paiements ou de façon plus indirecte, par l'intermédiaire d'un ciblage publicitaire.

18. Dans les années 1980-1990, le fondeur Intel équipe les compatibles PC et principalement IBM pour des machines avant tout dédiées à la bureautique, alors que Motorola va équiper Apple ainsi qu'Atari, très orienté vers les musiciens, et Amiga, plus orienté vers les graphistes.

19. Ce thème régulièrement abordé par la pensée critique est repris dans l'ouvrage du collectif Offensive Divertir pour dominer : la culture de masse contre les peuples, paru aux éditions L'échappée en 2010. Le second opus Divertir pour dominer 2 : la culture de masse toujours contre les peuples, dirigé par Cédric Biagini et Patrick Marcolini chez le même éditeur en 2019, y fait par contre une large place, signe des temps.

20. Pierre Bruno, Les Jeux vidéo, Paris, Syros, coll. « L'école des parents », 1993.

21. C'est la finalité de la thèse développée dans Cybernétique et société, annoncée dans la quatrième de couverture de l'édition du Seuil, 2014. Cybernétique et société peut être considéré comme une 
«mise au point» de Norbert Wiener, relative à la parution de Cybernetic en 1948. Certains y voient une attaque en règle de l'humanisme, d'autres la démonstration de l'intérêt du contrôle par les machines.

22. On distingue ici les hackers de code, les étudiants du MIT qui vont tenter d'accéder à la puissance de calcul fin des années 1950 début des années 1960 (voir « la logique du terminal ») et les ingénieurs et bricoleurs des années 1970 qui vont hacker les matériels existants pour initier l'informatique personnelle.

23. Il est fait ici allusion à ce qu'initialement dans les gros systèmes informatiques des débuts, la puissance de calcul était physiquement séparée des accessoires qui permettaient d'entrer les données, requêtes, programmes ou de récupérer les réponses post traitement. C'est en partie de la volonté de briser symboliquement ces murs à coup de hache que nait la notion de " hacker ", même si d'autres définitions seront établies par ces mêmes hackers dans leur manifeste de l'époque au MIT.

24. Par haut niveau, il faut comprendre haut niveau d'abstraction. Un langage de bas niveau est proche de la machine, comme l'écriture des circuits logique en électronique, a contrario un dispositif permettant d'écrire un programme en langage naturel serait de très haut niveau.

25. Pour donner une définition simple de ces termes, nous pouvons dire que l'art-game correspondrait à l'utilisation du jeu vidéo comme d'un médium artistique à part entière; le gameart à une utilisation des objets, techniques, et process du jeu vidéo dans une démarche d'art contemporain; le news-game à une utilisation du jeu vidéo comme support efficace pour rendre compte d'une situation à exposer, dénoncer, investir; le political-game a une utilisation plus engagée voir militante du support. Si ces définitions font l'objet de controverses, ce sont celles qui sont majoritairement admises dans le cadre universitaire, même si aujourd'hui la plupart des formes qui échappent au mainstream sont associée à l'art-game.

26. Le jeu casual, c'est le jeu occasionnel, pratiqué par ceux qui n'en revendiquent aucune expertise, qui n'y consacrent pas un temps spécifique. Par extension, une pratique qui se situe aux antipodes des pratiques immersives.

27. https://www.afjv.com/news/2567_le -design-des-jeux-freemium-pour-les-hardcoregamers.htm

28. Ces jeux, appelés auparavant Free to play, s'appuient sur un modèle économique basé sur une offre initiale gratuite qui parie sur des achats en ligne de différentes formes pour augmenter ou faciliter l'expérience de jeu. La transformation de quelques pourcentages de joueurs en acheteurs de ces microtransactions suffit à générer des profits colossaux, faisant de ce type de jeux les stars du R.O.I. (retour sur investissement). Les jeux sont accessibles librement mais l'ambiguïté est laissée sur le terme "gratuitement" - puisque le jeu, sous différentes formes devra bien être financé. Ce financement peut prendre la forme de publicités, mais aujourd'hui il s'agit plus souvent d'achats en cours de jeu, non obligatoires. Le modèle économique mise sur la conversion d'une partie des joueurs qui jouent gratuitement en joueurs qui payent. La dérive la plus décriée est celle du Pay to Win, qui fait du payeur un gagnant.

29. https://www.afjv.com/news/2640_le-design-des-jeux-freemium-pour-les-hardcoregamers.htm.

30. Marie-Aurélie Graff, «Entretien avec Alejandro Jodorowsky», 2008, URL: https:// www.ecranlarge.com/films/interview/901251-alejandro-jodorowsky-santa-sangre (consulté le 17 juin 2019).

31. Interview dans Métal Hurlant, $\mathrm{n}^{\circ} 107$.

32. Studio français qui sera parmi les plus importants des années 1990.

33. Daniel Ichbiah, Bâtisseurs de rêves: enquête sur le nouvel eldorado des jeux vidéo, F1rst, 1997, p. 192.

34. Avec L'Amerzone (1999) et ensuite les trois opus de Sibéria (2002- 2017), Benoit Sokal fait la démonstration du passage réussi d'un bédéaste au jeu vidéo, car sur l'essentiel il n'a rien cédé ou 
si peu aux contraintes de ce nouveau médium. À la sortie de L'Amerzone et plus encore dans la décennie qui va suivre, le jeu d'aventure de type point and click en 2D ou 2.5D est passé de mode, la 3D, les jeux d'action à la première personne ou les jeux en ligne multi-joueurs dominent le marché.

35. Initié par d'anciens membres de Lucas Arts, ce studio américain était l'un des meilleurs spécialistes du jeu d'aventure en point and click.

36. Genre de jeu vidéo qui utilise essentiellement la souris pour sélectionner des objets dans l'environnement ou pour déplacer son personnage. Souvent décrié comme ringard et en perte de vitesse dans les années 2000 il a retrouvé une jeunesse avec l'arrivée des tablettes et retrouve de plus en plus d'adeptes.

37. Marie-Aurélie Graff, « Entretien avec Alejandro Jodorowsky », op. cit.

38. Étienne Souriau, Les Différents modes d'existence, nouvelle édition préfacée par Bruno Latour et Isabelle Stengers, Paris, PUF, 2009.

39. Gilles Deleuze, "Qu'est-ce qu'un acte de création », in Pierre-André Boutang, L'Abécédaire de Gilles Deleuze, Montparnasse, 2004.

40. Gilles Deleuze prend exemple de la familiarité entre l'œuvre du cinéaste japonais notamment dans Les Sept samouraïs (1954) et la psychologie des personnages de Dostoïevski dont les enjeux dépassent les situations qu'ils vivent.

41. Jeu basé sur l'univers Pokémon qui utilise la réalité augmentée pour proposer une chasse au trésor avec l'apparition des pokémons sur les smartphones grâce à la géolocalisation.

42. Un modèle de casque de réalité virtuelle proposé sur plan par Google qui utilise des lentilles en plastique et un aimant. On en trouve montés à moins de deux euros.

43. La cinétose, communément appelée mal des transports, est un symptôme de la difficulté de s'adapter au mouvement et peut déclencher des nausées. C'est un véritable frein aux technologies de réalité virtuelle et plusieurs pistes sont explorées pour y remédier.

44. On trouvera une description et les brevets déposés à cette adresse: http:// www.mortonheilig.com/InventorVR.html.

45. Clin d'œil à la présence de cette machine dans l'œuvre de Jodorowsky, dans Dune dans la continuité de la mythologie depuis Dédale et Icare.

46. Marie-Aurélie Graff, « Entretien avec Alejandro Jodorowsky », op. cit.

47. Du pain et des jeux.

48. Tale of Tales est un studio de jeux vidéo belge s'attachant notamment à interroger les contes européens avec une vision critique. Leurs jeux sont poétiques, intimistes et complétement à contre-courant de la production mainstream. Avec par exemple l'adaptation du Petit Chaperon rouge en une fresque qui semble confronter les œuvres de Perrault, des frères Grimm et les analyses de Bettelheim.

\section{RÉSUMÉS}

Les Technopères, bande dessinée d'Alejandro Jodorowsky, voit son premier tome édité en 1998 aux Humanoïdes Associés. Il sera suivi de sept autres tomes qui composeront un récit se situant dans un univers initié par L'Incal et poursuivi par La Caste des Méta-barons qui, au travers de perspectives croisées, va enrichir un univers dystopique complexe. Une nouvelle fois, le propos de Jodorowsky s'en prend à la technologie, nouvelle religion d'un monde aliéné. Cette fois-ci c'est 
Albino que nous suivons au cours d'un voyage initiatique qui le mènera du rêve de faiseur de jeux vidéo au statut de technopère suprême. La description de la tension entre Albino et l'industrie du jeu vidéo dans cet univers est significative d'une analyse fine et documentée. La perspicacité de Jodorowsky sur l'orientation que va prendre ce médium dans les années qui vont suivre étonne par sa lucidité et fait regretter qu'il ne l'ait pas plus investi du point de vue de la création ou plutôt, comme il le dit, de la découverte. D'une certaine manière, la discussion développée dans cet article peut sonner comme un regret de cette non-rencontre mais il faut plutôt y lire une invitation.

Les Technopères, the techno-fathers, comics of Alejandro Jodorowsky had its first volume published in 1998, at the Humanoides Associés editing company. Afterwards, it is followed by seven other volumes composing a narrative situated in a universe beginning with L'Incal and carried on by La caste des Méta-barons which, through cross-cutting perspectives is nourishing a complex dystopic world. As usual, Jodorowsky's argument tackles the issue of technology, considered as a new religion in a context of alienation. In this story, we follow Albino through an initiatic travel, that will bring him from the dream of being a video game maker to the status of supreme technofather. The description of the tension between Albino and the video game industry as portrayed in this narrative, exhibits a well documented and subtle analysis. Jodorowsky's clear insight about the orientation that this media will take in the following years comes as a surprise for its lucidity and leaves a feeling of regret for not having invested more on this topic, from the creation - or discovery, as he would prefer to say- point of view. To some extent, the discussion developed in this paper may sound as a regret about this particular expectation, though we should rather see it as an invitation.

\section{AUTEUR}

\section{THIERRY SERDANE}

Maître de conférences en Arts Plastiques à l'université Paul-Valéry Montpellier 3. Il est responsable du Parcours Jeux vidéo au sein du Master Pratiques plastiques contemporaines et chercheur au RIRRA21. Ses axes de recherche s'articulent autour du jeu vidéo. Il s'intéresse autant à son processus de création qu'à son impact dans la société à partir d'une approche socio et techno-critique. 